\title{
Trends in Childhood Cancer in Kuwait: Data From the 2004-2017 Registry
}

Maha J. Bourusly ${ }^{1}$, Muna H. Burahma ${ }^{2}$, Nisreen Khalifa ${ }^{3}$, Hubert Motti ${ }^{3}$, Sahar Kaleefa ${ }^{3}$, Mohammad Adil $^{3}$, Suad Alanzi ${ }^{3}$, Medhat Alshazli ${ }^{4}$

1. Pediatric Hematology and Oncology, National Bank of Kuwait Specialized Hospital for Children, Sabah, KWT 2. Pediatrics, National Bank of Kuwait Specialized Hospital for Children, Sabah, KWT 3. Pediatric Oncology, National Bank of Kuwait Specialized Hospital for Children, Sabah, KWT 4. Pediatric Oncology, Ministry of Health, Kuwait City, KWT

Corresponding author: Maha J. Bourusly, m_bourusly@yahoo.com

\section{Abstract \\ Background and objective}

There is a lack of updated data regarding pediatric cancer in Kuwait, with no separate childhood cancer registry available in the country prior to this study. We aimed to determine the trends in different cancer types with respect to international statistics, in order to understand their nature and identify gaps in their diagnosis and management.

\section{Methods}

This retrospective study was based on data obtained from the first national childhood cancer registry of the National Bank of Kuwait (NBK) Specialized Hospital for Children, the only hospital that manages pediatric cancer patients in Kuwait. The registry included the data of all children with cancer in Kuwait from 2004 to 2017 and had complete data files.

\section{Findings}

The total number of patients in the childhood cancer registry was 1,387. A total of 1,009 files met our eligibility criteria. In 2017, the incidence of childhood leukemia was determined to be six per 100,000 people, and for other cancers, it was 12.6 per 100,000 people. The most common cancer was leukemia (457 patients), followed by lymphoma (141 patients), and brain tumors ( 92 patients). The majority of children received chemotherapy $(n=891,88.3 \%)$. Post induction or treatment, the majority of patients achieved complete remission $(n=790,78.3 \%)$. The overall survival rate of children with cancer was around $80 \%$. Major complications developed in $9 \%$ of patients.

\section{Conclusion}

Review began 01/13/202 Review ended 02/09/2021 Published 02/13/2021

\section{$\odot$ Copyright 2021}

Bourusly et al. This is an open access article distributed under the terms of the Creative Commons Attribution License CC-BY 4.0., which permits unrestricted CC-BY 4.0., which permits unrestricted
use, distribution, and reproduction in any medium, provided the original author and source are credited.

These findings highlight the importance of hospital-based cancer registries. Active data management programs are essential to monitor outcomes, measure the effectiveness of current practice, and improve the quality of care.

Categories: Pediatrics, Oncology, Hematology
Keywords: cancer registry, neoplasm, leukemia, childhood cancer, pediatric oncology

\section{Introduction}

Globally, more than 250,000 children are diagnosed with cancer each year. The incidence of pediatric malignancy has been on the rise for many reasons, including the improvement in the diagnosis and documentation of cancer patients [1]. According to the Central Statistical Bureau Annual Bulletin of Vital Statistics for Birth and Deaths, cancer was the second leading cause of death in adults in Kuwait between the years 2015-2017. Unfortunately, there are no specific mortality statistics for children below 14 years in Kuwait [2]. A published study based on the data of the Kuwaiti Cancer Registry (KCR) at the Kuwait Cancer Control Center (KCCCH) has reported that from 2010 to 2013, the highest five-year survival rate among patients aged more than 15 years old was in patients with lymphoma (96.3\%, 95\% CI: 91.4-100.0\%) followed by acute lymphocytic leukemia (ALL) (88.4\%, 95\% Cl: 80.6-96.2\%). However, between 2000 and 2013, the largest improvement in the survival rates of children was recorded among ALL patients, who showed a $12.3 \%$ increase in survival versus a $6.3 \%$ increase in lymphoma patients [3].

One of the main goals of the State of Kuwait 2035 Vision is the advancement of Kuwaiti healthcare [4]. Having a formal registry and documenting disease epidemiology and statistics play an important role in the planning and implementation of health plans and developing the public healthcare system [5].

Unfortunately, there is a lack of data and statistics on pediatric cancer in Kuwait, and data related to children with cancer are included in the adult national cancer registry. The objective of this study was to determine the trends and statistics related to different cancers affecting children in Kuwait.

\section{Materials And Methods \\ Study design and data collection}

This retrospective chart review used the data obtained from the national childhood cancer registry of the 
National Bank of Kuwait (NBK) Specialized Hospital for Children. All pediatric cancer patients in Kuwait are referred to this hospital and treated there. Data collection for the cancer registry started on May 12, 2016, and went on till December 2017, and included data of all children with cancer in Kuwait from January 2004 until December 2017. The datasheet used in the registry was finalized in accordance with the International Agency for Research on Cancer (IARC) guidelines and contained data regarding patient biography, investigations, clinical course, complications, and outcomes. The final data sheet was conceived after running a pilot study and reviewing registries from other countries and the Kuwait registry center. All children with cancer who attended the NBK Hospital and had complete data files were included in the study. Any patients with benign tumors or incomplete data files were excluded.

Entries were categorized according to the latest International Classification of Diseases (ICD), excluding stratification. The risk stratification of patients was based on the randomized United Kingdom National Acute Lymphoblastic Leukaemia (UKALL) 2003 study [6]. Investigators collected the data manually from the files of leukemia patients receiving treatment at the NBK Hospital from the year 2004 and from the files of patients with other cancers after 2005. Files from 2004 and 2005 belonged to leukemia patients only as other cancers were treated in adult hospitals prior to 2010, and hence their files were not available. After 2010, all children with cancer were treated at the NBK Hospital. Data from later dates (from 2010) were collected from electronic records, which were not available for earlier dates.

\section{Data analysis}

We analyzed the data collected to document the trends in childhood cancer in Kuwait. Data were processed and analyzed using SPSS Statistics for Windows, version 22 (IBM, Armonk, NY). Statistical analysis included quantitative descriptive analysis and summary statistics describing the frequency of diagnostic groups and subgroups of childhood cancer, in addition to survival data using the Kaplan-Meier survival curve.

\section{Study population}

Our study population included all children diagnosed with cancer in the state of Kuwait from 2004 to 2017 The details relating to the age of patients we studied were as follows: children under the age of 16 for the year 2017, children under the age of 15 from 2012 to 2017, and children under the age of 14 from 2004 to 2011. The difference in age range was due to the difference in the age of acceptance of patients at the NBK Hospital. Any child with an initial diagnosis of cancer in the state of Kuwait, regardless of nationality, is referred to the NBK Hospital for disease confirmation and treatment. Therefore, the cases included in this study represent the total number of childhood cancer patients in Kuwait, which amounted to a total of 1,387 After excluding duplicate files $(n=125)$ and files with insufficient data $(n=46)$, we analyzed the data from the remaining 1,009 files. Follow-up data were collected from the eligible records if they were available. The patients were followed up according to the routine practice followed at the NBK Hospital. Ethical approval was granted by the Standing Committee for Coordination of Health and Medical Research, and the Pediatric Departments Council at Kuwait's Ministry of Health; the need for informed consent was waived as the data used was secondary data, with no exposure of patient identity. The research protocol was conducted according to the principles of the Declaration of Helsinki.

\section{Results}

Out of 1,009 patient files, 457 were leukemia patients, and 552 were children with other malignancies. By reviewing the population details in the Kuwait census in the last two decades, we calculated the incidence rate for childhood leukemia as approximately six per 100,000 and for other cancers as nine per 100,000 for children below the age of 14 in 2011 . Table 1 shows the number of childhood cancer patients presenting to the NBK Hospital per year and the number of files retrieved. In 2017, the incidence rate of childhood leukemia was calculated as six per 100,000, and for other cancers as 12.6 per 100,000. However, the variability in incidence should be considered with caution because the population of Kuwait obtained from the national statistics center was for ages 0-14 years, and our study population included children aged 0-16 years. Moreover, we used the 2018 census to calculate the incidence rate, and so there may be a discrepancy between the actual rates and the rates we calculated. 


\section{Cureus}

\begin{tabular}{|c|c|c|c|c|c|}
\hline Year & New oncology patients & Files retrieved & New leukemia patients & Files retrieved & Total retrieved \\
\hline 2004 & 34 & 1 & 36 & 23 & 24 \\
\hline 2005 & 53 & 12 & 31 & 21 & 33 \\
\hline 2006 & 46 & 20 & 34 & 17 & 37 \\
\hline 2007 & 59 & 27 & 29 & 22 & 49 \\
\hline 2008 & 61 & 41 & 63 & 49 & 90 \\
\hline 2009 & 62 & 37 & 34 & 28 & 65 \\
\hline 2010 & 61 & 52 & 29 & 27 & 79 \\
\hline 2011 & 64 & 54 & 46 & 46 & 100 \\
\hline 2012 & 72 & 62 & 41 & 40 & 102 \\
\hline 2013 & 52 & 43 & 34 & 33 & 76 \\
\hline 2014 & 67 & 51 & 41 & 41 & 92 \\
\hline 2015 & 74 & 67 & 31 & 28 & 95 \\
\hline 2016 & 68 & 64 & 43 & 39 & 103 \\
\hline 2017 & 77 & 66 & 45 & 44 & 110 \\
\hline
\end{tabular}

TABLE 1: Number of childhood cancer patients presenting to the NBK Hospital per year and the number of files retrieved

NBK: National Bank of Kuwait

\section{Patient characteristics}

There were 588 males and 421 females, with ages ranging from one month to 16 years; the mean age was six years. There were 593 Kuwaitis and 416 non-Kuwaitis of 28 other nationalities (Figure 1). Only 7\% (70/939) of the patients had known genetic diseases and the most frequent genetic disease was Down syndrome ( $n=37,3.7 \%$ of all population), followed by hereditary retinoblastoma ( $n=12,2 \%$ of the population). Previous medical history was reported in 92 children, such as diabetes mellitus, cardiac diseases, central nervous system (CNS) diseases, and others. The most frequent medical condition was cardiac diseases $(n=27)$ followed by CNS diseases $(n=18)$ and respiratory diseases $(n=15)$.

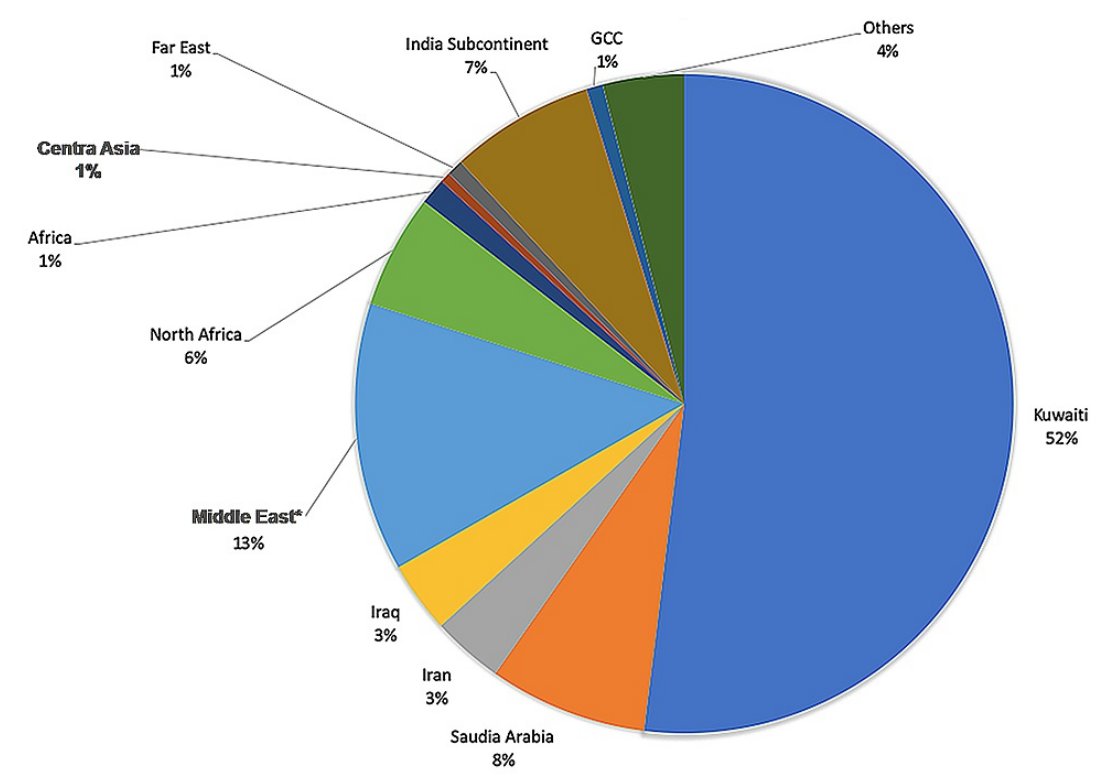

FIGURE 1: Nationalities of children with cancer presenting to the NBK Hospital in Kuwait between 2004-2017

*Middle East: all middle eastern countries excluding Saudi Arabia, Iran, Iraq, and Kuwait

NBK: National Bank of Kuwait; GCC: Gulf Cooperation Council 
A positive family history of cancer was recorded in 298 files, either in a first-degree relative ( $n=33,3.3 \%)$, second-degree relative $(n=110,10.9 \%)$, or an extended family member $(n=155,15.4 \%)$. The level of education of parents was assessed; $35.7 \%(n=357)$ of the parents were educated to the level of high school. The majority of parents had intermediate education (no high school certification) ( $\mathrm{n}=552,54.7 \%$ ); in a few families, both parents did not receive any formal education $(\mathrm{n}=12,1.2 \%)$, and data were missing in 88 files. The number of siblings was mentioned in 619 files (mean: 2.86; range: 0-22). Almost half of the patients were in preschool $(49 \%, \mathrm{n}=479)$, and $46.8 \%$ were at school, in one of the three stages of school education $(\mathrm{n}=470)$. Unfortunately, $4.2 \%(\mathrm{n}=42)$ of children were school-aged but did not receive any formal education.

\section{Diagnosis}

Positive histology samples were used for diagnosis in $48.7 \%$ of patients $(n=491)$, and positive laboratory tests or markers were used for diagnosis in $46.1 \%$ of patients $(n=465)$. Immune phenotyping was frequently used as a diagnostic tool for leukemia but rarely used in other cancers. The most frequently recurrent immunephenotyping values were CD10, CD19, CD45, CD34, and HLA-DR.

Cytogenetics were used for the diagnosis and prediction of the prognosis of childhood leukemia. Normal karyotypes with no aberrations were detected in 120 children, while 57 children showed normal karyotypes with detected aberrations. Ninety-nine children showed hyperdiploidy; while five children had hypoploidy. Cytogenetic analysis was not recorded in 597 patients (mainly in children with cancers other than leukemia).

\section{Cancer types and disease risk}

For the sake of convenience, we divided cancers into two categories: solid tumors (including brain tumors, lymphomas, and bone, renal, and other tumors), and leukemia. The main primary sites of cancer in nonleukemia patients were the abdomen and pelvis (34\%) followed by the head and neck (33\%). In the solid tumor group, $50.7 \%$ of patients $(n=280)$ had localized tumors, $16 \%(n=92)$ had distant metastasis, and $16.1 \%$ $(n=89)$ had localized tumors with direct extension.

The most prevalent cancer was leukemia, followed by lymphoma and brain tumors. The most common brain tumor in our registry was glioma (52.7\%), followed by medulloblastoma (30.7\%), anaplastic ependymoma (8.7\%), and brain and spine tumors (6.5\%). The most prevalent leukemia was ALL (80\%), followed by acute myeloblastic leukemia (AML, 16.6\%) and chronic myeloid leukemia (CML, 3\%). Children with Down syndrome developed leukemia $(n=35)$ and other solid tumors $(n=2)$. Fourteen children with Down syndrome had AML, and 21 had ALL.

Risk stratification was performed, and patients were divided according to their risk into high-risk (30.8\%, $\mathrm{n}=311)$, intermediate-risk (33\%, $\mathrm{n}=333)$, and low-risk $(29.1 \%, \mathrm{n}=294)$ categories. There were 71 children with undetermined risk, or where the calculation of risk was non-applicable. The disease risk changed in certain children, mostly in children with leukemia for many reasons; either due to minimal residual disease (MRD) results $(n=61)$, end of induction bone marrow results $(n=12)$, or due to non-specific reasons $(n=5)$.

\section{Treatment and complications}

Surgical procedures were performed for 510 patients; 100 patients underwent a second surgical procedure, and 13 underwent a third surgical procedure (Table 2). Post-surgical complications were reported in $4.1 \%$ $(n=21)$ of patients.

\begin{tabular}{|c|c|c|c|}
\hline Type of surgery & First surgical procedure, $\mathrm{n}(\%)$ & Second surgical procedure, $\mathrm{n}(\%)$ & Third surgical procedure, $\mathrm{n}(\%)$ \\
\hline Biopsy only & $255(50 \%)$ & $11(2.2 \%)$ & $2(0.4 \%)$ \\
\hline Partial resection & $79(15.5 \%)$ & $30(5.9 \%)$ & $3(0.6 \%)$ \\
\hline Radical resection & $170(33.3 \%)$ & $54(10.6 \%)$ & $5(1 \%)$ \\
\hline Metastatectomy & $2(0.4 \%)$ & $5(1 \%)$ & $2(0.4 \%)$ \\
\hline Other & $1(0.2 \%)$ & $0(0 \%)$ & $1(0.2 \%)$ \\
\hline Total & 507 (99.4\%) & $100(19.6 \%)$ & $13(2.5 \%)$ \\
\hline Missing values & $3(0.6 \%)$ & $41(80.4 \%)$ & $497(97.5 \%)$ \\
\hline Total & $510(100 \%)$ & $510(100 \%)$ & $510(100 \%)$ \\
\hline
\end{tabular}

TABLE 2: Different surgical procedures in children with cancer

Out of the 92 children who were diagnosed with brain tumors, 86 children underwent surgical procedures. Children with glioma were the group most likely to have a surgical procedure (42/92, 87.5\%) (Table 3). 


\section{Cureus}

\begin{tabular}{|c|c|c|c|}
\hline Brain tumor & Conservative approach, n (\%) & Surgical approach, $\mathbf{n}(\%)$ & Total, n (\%) \\
\hline Atypical rhabdoid teratoid brain tumor & $0(0 \%)$ & $1(100 \%)$ & $1(100 \%)$ \\
\hline Glioma & $6(12.5 \%)$ & 42 (87.5\%) & $48(100 \%)$ \\
\hline Medulloblastoma & $0(0 \%)$ & $28(100 \%)$ & $28(100 \%)$ \\
\hline Anaplastic ependymoma & $0(0 \%)$ & $8(100 \%)$ & $8(100 \%)$ \\
\hline Sialoblastoma & $0(0 \%)$ & $1(100 \%)$ & $1(100 \%)$ \\
\hline Other tumors of the brain and spine & $0(0 \%)$ & $6(100 \%)$ & $6(100 \%)$ \\
\hline Total & $6(6.5 \%)$ & $86(93.5 \%)$ & $92(100 \%)$ \\
\hline
\end{tabular}

TABLE 3: Surgery versus conservative approach for different types of brain tumors

Radiation therapy was reported in $13.3 \%$ of patients $(n=133 / 1,009)$ and was well tolerated in $82 \%$ of patients ( $n=109)$, while $18.1 \%(n=24 / 133)$ developed post-radiotherapy complications. The type of radiotherapy they received was either 3D ( $n=15 / 133,11.3 \%)$, conformal $(n=95 / 133,71.4 \%)$, intensity-modulated radiation therapy $(n=14 / 133,10.5 \%)$, or proton therapy $(n=9 / 133,6.8 \%)$. Radiotherapy was used as radical $(n=60 / 133$, $45 \%)$, palliative ( $n=11 / 133,8.3 \%)$, or adjuvant $(n=62 / 133,46 \%)$ treatment. Only five leukemia patients received radiotherapy. Table 4 presents the types of radiation received by children with solid cancers.

\begin{tabular}{|c|c|c|c|c|c|c|}
\hline \multicolumn{2}{|l|}{ Brain tumor } & 3D & Conformal & IMRT & Proton & Total \\
\hline \multirow{2}{*}{ Glioma } & $\mathrm{N}$ & 1 & 15 & 1 & 1 & 18 \\
\hline & $\%$ & $5.60 \%$ & $83.30 \%$ & $5.60 \%$ & $5.60 \%$ & $100 \%$ \\
\hline \multirow{2}{*}{ Medulloblastoma } & $\mathrm{N}$ & 0 & 19 & 1 & 2 & 22 \\
\hline & $\%$ & $0 \%$ & $86.40 \%$ & $4.50 \%$ & $9.10 \%$ & $100 \%$ \\
\hline \multirow{2}{*}{ Anaplastic ependymoma } & $\mathrm{N}$ & 1 & 5 & 0 & 1 & 7 \\
\hline & $\%$ & $14.30 \%$ & $71.40 \%$ & $0 \%$ & $14.30 \%$ & $100 \%$ \\
\hline \multirow{2}{*}{ Sialoblastoma } & $\mathrm{N}$ & 0 & 1 & 0 & 0 & 1 \\
\hline & $\%$ & $0 \%$ & $100 \%$ & $0 \%$ & $0 \%$ & $100 \%$ \\
\hline \multirow{2}{*}{ Other tumors of the brain and spine } & $\mathrm{N}$ & 1 & 0 & 0 & 0 & 1 \\
\hline & $\%$ & $100 \%$ & $0 \%$ & $0 \%$ & $0 \%$ & $100 \%$ \\
\hline \multirow{2}{*}{ Total } & $\mathrm{N}$ & 3 & 40 & 2 & 4 & 49 \\
\hline & $\%$ & $6.10 \%$ & $81.60 \%$ & $4.10 \%$ & $8.20 \%$ & $100 \%$ \\
\hline
\end{tabular}

TABLE 4: Type of radiation received by children with solid cancers

IMRT: intensity-modulated radiation therapy

The majority of children received chemotherapy ( $\mathrm{n}=891 / 1,009,88.3 \%)$. The chemotherapy protocols received were either the British protocol ( $\mathrm{n}=426 / 891,42 \%$ ) or the International Society of Pediatric Oncology (SIOP) protocol ( $n=315 / 891,31 \%)$. Information was missing regarding the chemotherapy protocol used in 118 children (Table 5). Out of 92 children who had brain tumors, 57 children received chemotherapy (Table 6 ). 


\section{Cureus}

\begin{tabular}{|c|c|c|}
\hline Name of the chemotherapy protocol & Number of patients & Percentage \\
\hline SIOP & 318 & $31.50 \%$ \\
\hline UK-MRC & 426 & $42.20 \%$ \\
\hline FRALLE & 15 & $1.50 \%$ \\
\hline COG & 71 & $7 \%$ \\
\hline BFM & 15 & $1.50 \%$ \\
\hline Undefined/unspecified & 26 & $2.60 \%$ \\
\hline Other & 20 & $20 \%$ \\
\hline Total & 891 & $88.30 \%$ \\
\hline Missing & 118 & $11.70 \%$ \\
\hline Total population & 1,009 & $100 \%$ \\
\hline
\end{tabular}

TABLE 5: Different chemotherapy protocols received by children in our study

SIOP: International Society of Pediatric Oncology; UK-MRC: United Kingdom-Medical Research Council; FRALLE: French Acute Lymphoblastic Leukemia; COG: Children's Oncology Group; BFM: Berlin-Frankfurt-Munich

\begin{tabular}{|c|c|c|c|c|}
\hline \multicolumn{2}{|l|}{ Brain tumor } & No chemotherapy & Chemotherapy & Total \\
\hline \multirow{2}{*}{ Atypical rhabdoid teratoid brain tumor } & $\mathrm{N}$ & 0 & 1 & 1 \\
\hline & $\%$ & $0 \%$ & $100 \%$ & $100 \%$ \\
\hline \multirow{2}{*}{ Glioma } & $\mathrm{N}$ & 25 & 23 & 48 \\
\hline & $\%$ & $52.10 \%$ & $47.90 \%$ & $100 \%$ \\
\hline \multirow{2}{*}{ Medulloblastoma } & $\mathrm{N}$ & 2 & 26 & 28 \\
\hline & $\%$ & $7.10 \%$ & $92.90 \%$ & $100 \%$ \\
\hline \multirow{2}{*}{ Anaplastic ependymoma } & $\mathrm{N}$ & 4 & 4 & 8 \\
\hline & $\%$ & $50 \%$ & $50 \%$ & $100 \%$ \\
\hline \multirow{2}{*}{ Sialoblastoma } & $\mathrm{N}$ & 0 & 1 & 1 \\
\hline & $\%$ & $0 \%$ & $100 \%$ & $100 \%$ \\
\hline \multirow{2}{*}{ Other tumors of the brain and spine } & $\mathrm{N}$ & 4 & 2 & 6 \\
\hline & $\%$ & $66.70 \%$ & $33.30 \%$ & $100 \%$ \\
\hline \multirow{2}{*}{ Total } & $\mathrm{N}$ & 35 & 57 & 92 \\
\hline & $\%$ & $38 \%$ & $62 \%$ & $100 \%$ \\
\hline \multicolumn{5}{|c|}{ TABLE 6: Number of patients who received chemotherapy for different tumors } \\
\hline \multicolumn{5}{|c|}{$\begin{array}{l}\text { Almost one-third of the patients developed toxicities related to chemotherapy ( } \mathrm{n}=317,35 \%) \text {, while } 64.4 \% \\
(\mathrm{n}=574) \text { did not report any toxicity; } 12 \% \text { developed late complications in one or more systems related to } \\
\text { therapy, and } 60 \%(\mathrm{n}=540 / 891) \text { reported chemotherapy-related complications. Constitutional symptoms were } \\
\text { reported in } 13.1 \% \text { of the patients }(\mathrm{n}=117) \text { such as loss of appetite, weight loss, and general fatigue. The most } \\
\text { common early side effects were febrile neutropenia or infections, which were found in } 44.1 \% \text { of the patients } \\
(\mathrm{n}=393) \text {. A detailed presentation of the early complications is given in Table } 7 \text {. }\end{array}$} \\
\hline
\end{tabular}




\begin{tabular}{|c|c|c|c|c|c|c|c|c|c|}
\hline & Infection/F\&FN & Allergic/immune-related & BM/BLD & Gl & Metabolic & Ophthalmic & Skin-related & Pain & Renal \\
\hline Yes & 393 (44.1\%) & $95(10.7 \%)$ & $202(22.7 \%)$ & 104 (11.7\%) & 106 (11.9\%) & 12 (1.3\%) & $23(2.6 \%)$ & $27(3 \%)$ & 14 (1.5\%) \\
\hline No & $498(55.9 \%)$ & $798(89.3 \%)$ & $689(77.3 \%)$ & $787(88.13 \%)$ & $783(88 \%)$ & $879(98.7 \%)$ & $868(97.4 \%)$ & $864(97 \%)$ & $877(98 \%)$ \\
\hline
\end{tabular}

TABLE 7: Early side effects of chemotherapy in our study population

F\&FN: fever and febrile neutropenia; BM/BLD: bone marrow/blood; Gl: gastrointestinal

Late treatment complications were reported in $12.8 \%$ of patients $(n=129)$ (Table 8$)$. Late treatment-related complications of the CNS were most often neurological (sensory or motor complications) $(n=51$, $39.5 \%$ ), followed by neurocognitive or psycho-social complications.

\begin{tabular}{|c|c|c|}
\hline Type of complication & Number of patients & Percentage \\
\hline Cardiovascular system & 9 & $0.9 \%$ \\
\hline Central nervous system & 36 & $3.6 \%$ \\
\hline Digestive system & 4 & $0.4 \%$ \\
\hline Endocrine system & 25 & $2.5 \%$ \\
\hline Immune system & 3 & $0.3 \%$ \\
\hline Musculoskeletal & 21 & $2.1 \%$ \\
\hline Reproductive & 2 & $0.2 \%$ \\
\hline Respiratory & 2 & $0.2 \%$ \\
\hline Special senses & 22 & $2.2 \%$ \\
\hline Urinary system & 5 & $0.5 \%$ \\
\hline Total & 129 & $12.8 \%$ \\
\hline Missing & 880 & $87.2 \%$ \\
\hline Total & 1,009 & $100 \%$ \\
\hline
\end{tabular}

TABLE 8: Late side effects of chemotherapy in our study population

\begin{abstract}
Disease status and survival
Post induction or treatment, the majority of patients went into remission ( $\mathrm{n}=790,78.3 \%)$, while some had partial remission $(n=35,3.6 \%)$; some developed progressive disease $(n=37,3.7 \%)$ or relapsed $(n=56,5.6 \%)$. Disease status was followed up at six months, 18 months, and 24 months (Table 9). The majority of children who were lost to follow-up at six months were Kuwaitis (Table 10); $11 \%$ of the children were lost to followup; they were mainly of preschool age (zero to four years) followed by children of primary school age (five to nine years).
\end{abstract}

\begin{tabular}{|c|c|c|c|c|c|c|c|c|c|c|}
\hline \multirow[t]{2}{*}{ Status } & \multicolumn{2}{|c|}{ Post induction } & \multicolumn{2}{|c|}{6 months } & \multicolumn{2}{|c|}{12 months } & \multicolumn{2}{|c|}{18 months } & \multicolumn{2}{|c|}{24 months } \\
\hline & $\mathrm{N}$ & $\%$ & $\mathrm{~N}$ & $\%$ & $\mathrm{~N}$ & $\%$ & $\mathrm{~N}$ & $\%$ & $\mathrm{~N}$ & $\%$ \\
\hline Complete remission & 790 & $78.3 \%$ & 793 & $78.6 \%$ & 761 & $75.4 \%$ & 703 & $69.7 \%$ & 665 & $65 \%$ \\
\hline Partial remission & 35 & $3.6 \%$ & 29 & $2.9 \%$ & 23 & $2.3 \%$ & 18 & $1.8 \%$ & 14 & $1.4 \%$ \\
\hline Progressive disease & 37 & $3.7 \%$ & 36 & $3.6 \%$ & 16 & $1.6 \%$ & 11 & $1.1 \%$ & 8 & $0.8 \%$ \\
\hline Relapse & 56 & $5.6 \%$ & 22 & $2.2 \%$ & 22 & $2.2 \%$ & 18 & $1.8 \%$ & 19 & $1.9 \%$ \\
\hline Missing data & 90 & $8.9 \%$ & 116 & $11.5 \%$ & 163 & $16.2 \%$ & 226 & $22.4 \%$ & 266 & $26.4 \%$ \\
\hline
\end{tabular}

TABLE 9: Disease status of children with cancer post induction or treatment and at follow-up 


\section{Cureus}

\begin{tabular}{|c|c|c|}
\hline Patient nationality & Number of patients & Percentage \\
\hline Kuwaiti & 68 & $58.60 \%$ \\
\hline Non-defined & 7 & $6 \%$ \\
\hline Saudi Arabian & 4 & $3.40 \%$ \\
\hline Egyptian & 9 & $7.70 \%$ \\
\hline Syrian & 6 & $5.10 \%$ \\
\hline Lebanese & 1 & $0.80 \%$ \\
\hline Other GCC countries & 1 & $0.80 \%$ \\
\hline India & $r$ & $6 \%$ \\
\hline Bangladesh & 1 & $0.86 \%$ \\
\hline Iran & 1 & $0.86 \%$ \\
\hline Iraq & 2 & $1.70 \%$ \\
\hline Others & 6 & $5.10 \%$ \\
\hline Missing data & 3 & $2.50 \%$ \\
\hline Total & 116 & $100 \%$ \\
\hline
\end{tabular}

TABLE 10: Nationalities of children with cancer who were lost to follow-up

The overall survival graph shows the survival of children with cancer in Kuwait to be around $80 \%$ (Figure $2 A$ ). The overall survival rate for leukemia in comparison with other cancers was around $70 \%$, and the overall survival rate for other cancers was around $80 \%$ (Figure $2 B$ ). The overall five-year survival rate of cancers excluding leukemia was around $80 \%$ (Figure 2 C), and the 10 -year survival rate was almost the same.

Most childhood brain tumors were low-grade gliomas and medulloblastomas. The overall five-year survival rate of brain tumors in children in Kuwait was $60 \%$. This survival curve remains at a plateau at 10 years (Figure $2 D$ ). However, this survival curve does not necessarily reflect the scope of brain tumor disease in Kuwait, as some cases skip the registration process at our center.

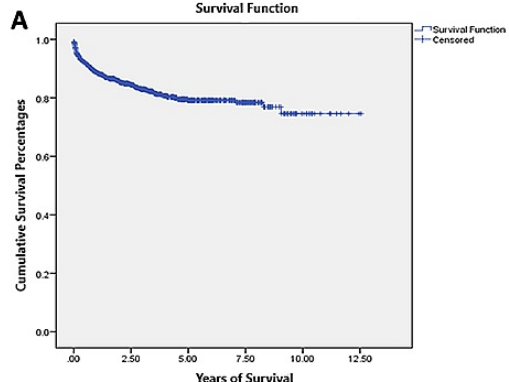

C
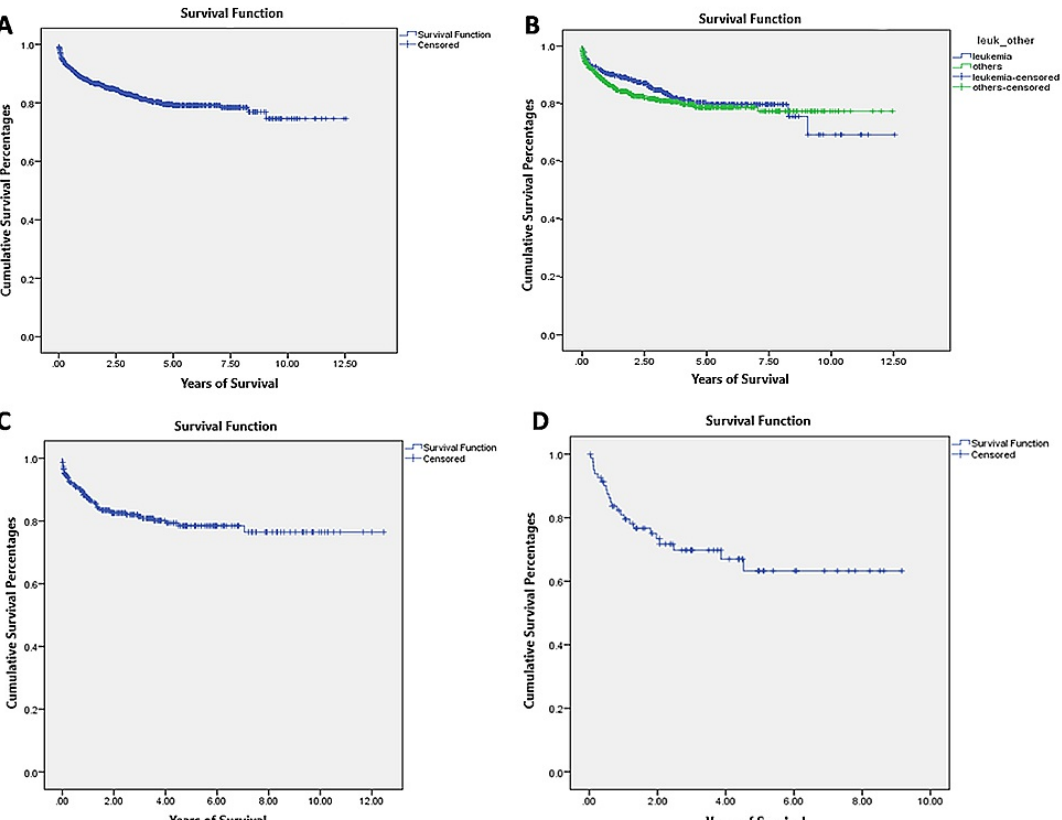

D

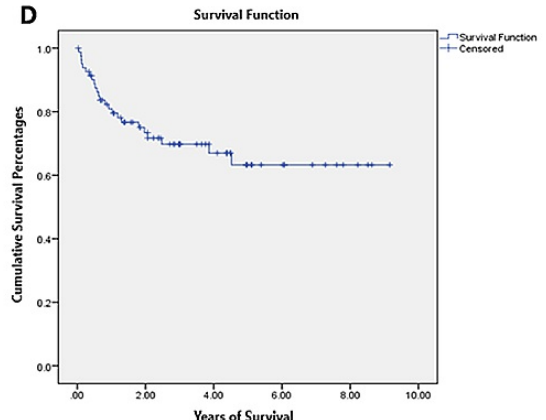

FIGURE 2: Survival curves of childhood cancer patients in Kuwait

A) overall survival rate of children with cancer in Kuwait; B) overall survival rate of children with leukemia in comparison with other cancers in Kuwait; C) overall survival of children with cancers excluding leukemia in Kuwait; D) overall survival rate of children with brain cancer in Kuwait 


\section{Cureus}

\section{Major problems related to disease and treatment}

Major complications occurred in $9 \%$ of patients either due to disease itself or its management. These problems were as follows: relapse, fatal sepsis, obesity, secondary cancer, or neurological sequelae. Relapse occurred in 53 children either within two years, five years, or even after five years of treatment. Fourteen children were lost to fatal infections, and four children reported secondary cancer (Table 11), i.e., 1:252 developed secondary cancer. Some children required second-line treatment $(\mathrm{n}=128,12.7 \%)$. Bone marrow transplant was the only treatment option in $6.3 \%$ of children $(n=64)$ and some children $(2.8 \%, n=28)$ had persistent or aggressive diseases or developed a debilitative state and required palliative care. Salvage treatment was the main option in $4.8 \%(n=48 / 128)$ of children who required second-line treatment, while targeted treatment was the treatment option for $0.8 \%(n=8 / 128)$ of children. The majority of children were alive and well when last seen. Unfortunately, 10\% passed away, 11\% were lost to follow-up, and 3\% were left with a permanent disability (Table 12).

\begin{tabular}{|c|c|c|}
\hline Major complication & Number of patients & Percentage \\
\hline Obesity & 3 & $0.30 \%$ \\
\hline Severe weight loss & 2 & $0.20 \%$ \\
\hline Major neurological sequelae & 15 & $1.50 \%$ \\
\hline Hematological relapse after 2 years & 37 & $3.70 \%$ \\
\hline CNS relapse after 2 years & 2 & $0.20 \%$ \\
\hline Relapse within 5 years of stopping treatment & 10 & $1 \%$ \\
\hline Relapse after 5 years of stopping treatment & 3 & $0.30 \%$ \\
\hline Fatal sepsis & 14 & $1.40 \%$ \\
\hline Secondary cancer & 4 & $0.40 \%$ \\
\hline Total & 90 & $8.90 \%$ \\
\hline Missing values & 919 & $91.10 \%$ \\
\hline Total population & 1,009 & $100 \%$ \\
\hline
\end{tabular}

TABLE 11: Major complications in children with cancer in Kuwait

CNS: central nervous system

\begin{tabular}{|c|c|c|}
\hline Status at last contact & Number of patients & Percentage \\
\hline Living and well & 770 & $76.30 \%$ \\
\hline Deceased & 95 & $9.40 \%$ \\
\hline Living and debilitated & 31 & $3.10 \%$ \\
\hline Lost to follow-up & 113 & $11.20 \%$ \\
\hline Total & 1,009 & $100 \%$ \\
\hline
\end{tabular}

TABLE 12: Status at last contact of children with cancer in Kuwait

\section{Discussion}

Cancer is a major health problem in terms of morbidity and mortality worldwide and especially in developing countries [7]. The incidence rates of childhood cancer have been increasing, and so have the survival rates [8]. Although there has been an improvement in the five-year survival rates, childhood cancer is the second leading cause of death after accidents in developed countries [9]. Moreover, 30\% of childhood cancer deaths are due to hematological malignancies [10].

There are ethnic, racial, and geographic variations in the incidence of childhood cancer and survival worldwide [11]. In our study, almost $60 \%$ of patients were Kuwaitis, and the rest were non-Kuwaitis of different nationalities. The incidence rate we calculated was similar to the incidence rate in other countries $[12,13]$. Furthermore, incidence rates were higher in males compared to females, which is consistent with all international statistics [14]. 
With regard to cancer risk factors, the most common genetic disease reported was Down syndrome [15]. This may be explained by the fact that Kuwaiti women choose to have children throughout their fertile years, which may increase the incidence of Down syndrome in their later pregnancies. Additionally, consanguineous marriages are prevalent among the Kuwaiti population, which poses an added risk [16]. Past medical history was not common, and the most commonly reported disease was a cardiac disease. This can be explained by the recent studies linking cancer with cardiac diseases, as congenital cardiac diseases have been associated with the development of cancer [17]. Our findings of the prevalence of a positive family history of cancer were similar to other studies, and the majority of the positive family history was related to members of patients' extended family [18,19].

The majority of parents had a higher education degree, which does not match the general description of the population in Kuwait, where the majority have intermediate education only [20]. We hypothesize that parents who have intermediate education are mostly expats and do not have the means to support families in Kuwait, and so their children do not contribute to Kuwait's childhood cancer statistics [2]. The majority of families in this study were middle- or high-income families, and members were well-educated, and this is consistent with the studies that have associated the parents' education and socioeconomic status with long-term survival, especially for cancers that require long-term treatment [21].

According to a Global Cancer Observatory (GLOBOCAN) estimation of cancer rates in 2018, leukemia was the most common childhood malignancy worldwide, followed by malignancies of the brain and nervous system, non-Hodgkin lymphomas, renal tumors, and Hodgkin lymphomas. However, the rank and outcome of the most common cancer types varied among countries [22]. Our results were almost similar to other registries in the region [23].

The incidence of leukemia we calculated was higher than the general international incidence [24]. This is probably due to the fact that we could not retrieve the files for the children with oncology diseases and only retrieved the files for leukemia patients for the year 2004. Some files from 2004 and the following five years were not brought to our center and hence were lost from the registry. The distribution and five-year survival rates of different types of leukemia were in line with international statistics $[8,25]$. The overall survival for leukemia has improved; the five-year survival was around 80\%, and the overall 10-year survival for leukemia was $70 \%$.

The second most prevalent tumor in children in Kuwait was lymphoma, which is not the case elsewhere where brain tumors have a higher incidence than lymphoma. One reason could be the overlap of lymphomas and leukemia with regard to the clinical presentation and course of the disease, as we have recorded a few children with lymphoma who presented with bone marrow infiltration. Many children were diagnosed in the pediatric neurosurgery department and had surgeries, or traveled abroad seeking specialized treatment for complicated cases, or died without showing up at our center. Similar problems have been documented in other studies [26]. Nevertheless, more research is needed to explain the increased incidence of lymphoma. The survival rate of lymphoma was high, which is in keeping with international statistics, and this reflects the improvements in the treatment methods of Hodgkin and non-Hodgkin lymphoma.

Toxicities related to chemotherapy developed in around a third of the patients. One of the least reported complications was pain (3\%), it could be attributed to underreporting or the sound pain management protocol that was developed in 2011. Endocrine problems were rarely reported, although steroid-induced hyperglycemia is frequently witnessed in clinical practice. One explanation is that these complications were mistakenly counted with metabolic/laboratory complications. Late treatment complications were also reported in a small number of patients, which contrasts with other studies [27]. Hence, we believe they were underreported.

The majority of children went into remission after treatment, and this is consistent with international statistics [28]. The number of children in remission at presentation was higher than at 24 months as some children were lost to follow-up as they had either traveled abroad for further treatment or had relapsed and were referred to another specialty for further treatment. The number of patients who developed secondary cancer in our study was higher than the number documented in other studies [29]. This mandates creating an algorithm to identify children at risk of secondary cancer to avoid adverse outcomes.

The survival rate for all children with cancer in Kuwait was high, which is in line with international statistics, and so was the incidence in comparison to survival rates [1,30]. This may be a good reflection of the services provided, but it does not address the survival rates of different cancers. Our study population had a heterogeneous genetic make-up, and this might have affected cancer prevalence and management as survival differs among different types of cancer. In addition, many of our patients with the same disease received different treatment protocols according to the treatment center, and this might have influenced our results.

This project has transformed the way we gather information in our center. We have worked on standardizing the data collection process on the registration of new patients to prevent the obstacles we faced in our study.

\section{Limitations}

The data collection process for the childhood cancer registry was not comprehensive. The dated manual system and the transfer of patients between hospitals prior to 2010 made it impossible to get all the required data from files from other hospitals. Many files were excluded from the study because they lacked essential information, and almost $11 \%$ of enrolled children were lost to follow-up, which may have affected the survival curve. 


\section{Conclusions}

Based on our findings, the trends in childhood cancer statistics in Kuwait share some similarities with international statistics. However, the incidence of leukemia was higher than the international rates, and the second most common cancer was lymphoma, rather than brain tumors. Survival rates were as high as in other countries, which reflects the good standard of care in Kuwait. A hospital-based cancer registry and active data management program are required to explore the difference in incidence rates in Kuwait compared to other countries and measure the effectiveness of specific interventions to improve the quality of care. Funding registries such as ours would help in the improvement of childhood survival through continuous assessment and auditing of the services provided.

\section{Additional Information \\ Disclosures}

Human subjects: Consent was obtained or waived by all participants in this study. National Bank of Kuwait Hospital Ethics Committee issued approval N/A. The study's protocol was approved by the ethics committee of the National Bank of Kuwait Hospital. Animal subjects: All authors have confirmed that this study did not involve animal subjects or tissue. Conflicts of interest: In compliance with the ICMJE uniform disclosure form, all authors declare the following: Payment/services info: The work was supported by the Kuwait Foundation for the Advancement of Sciences (KFAS) - 01 P116-13MK. Financial relationships: All authors have declared that they have no financial relationships at present or within the previous three years with any organizations that might have an interest in the submitted work. Other relationships: All authors have declared that there are no other relationships or activities that could appear to have influenced the submitted work.

\section{Acknowledgements}

We would like to thank all the people who contributed to this project for their hard work, effort, and dedication; researchers: Mona Bourhama, Nisreen Khalifa, Hubert Motti, Sahar Kaleefa, and Muhammed Adil; statisticians: Suad Alenzi and Medhat Alshazli; assistant technicians: Mrs. Jomol Johnson and Miss Rehab Alkhaldi.

\section{References}

1. Steliarova-Foucher E, Fidler MM, Colombet M, et al.: Changing geographical patterns and trends in cancer incidence in children and adolescents in Europe, 1991-2010 (Automated Childhood Cancer Information System): a population-based study. Lancet Oncol. 2018, 19:1159-1169. 10.1016/S1470-2045(18)30423-6

2. Population estimates in Kuwait by age, nationality and sex . (2020). Accessed: February 12, 2021: https://csb.gov.kw/Pages/Statistics_en?ID=67\&ParentCatID=1.

3. Alawadhi E, Al-Awadi A, Elbasmi A, Coleman MP, Allemani C: Cancer survival by stage at diagnosis in Kuwait: a population-based study. J Oncol. 2019, 2019:8463195. 10.1155/2019/8463195

4. Kuwait National Development Plan. (2019). Accessed: February 12, 2021: https://newkuwaitsummit.com/kndp\#: :text=The\%20Kuwait\%20National\%20Development\%20Plan,focus\%20for\%20investment\%:

5. Leal YA, Fernández-Garrote LM, Mohar-Betancourt A, Meneses-García A: The importance of registries in cancer control. Salud Publica Mex. 2016, 58:309-316. 10.21149/spm.v58i2.7802

6. Vora A, Goulden N, Wade R, et al.: Treatment reduction for children and young adults with low-risk acute lymphoblastic leukaemia defined by minimal residual disease (UKALL 2003): a randomised controlled trial. Lancet Oncol. 2013, 14:199-209. 10.1016/S1470-2045(12)70600-9

7. Haileamlak A: The challenge of childhood cancer in developing countries . Ethiop J Health Sci. 2016, 26:199200.

8. Smith MA, Seibel NL, Altekruse SF, et al.: Outcomes for children and adolescents with cancer: challenges for the twenty-first century. J Clin Oncol. 2010, 28:2625-2634. 10.1200/JCO.2009.27.0421

9. Al-Asadi JN, Ibrahim SJ: Childhood cancer in Basrah, Iraq during 2012-2016: incidence and mortality . Asian Pac J Cancer Prev. 2018, 19:2337-2341. 10.22034/APJCP.2018.19.8.2337

10. Gavin A, Rous B, Marcos-Gragera R, et al.: Towards optimal clinical and epidemiological registration of haematological malignancies: guidelines for recording progressions, transformations and multiple diagnoses. Eur J Cancer. 2015, 51:1109-1122. 10.1016/j.ejca.2014.02.008

11. Janitz AE, Campbell JE, Pate A, Anderson A, McNall-Knapp R: Racial, ethnic, and age differences in the incidence and survival of childhood cancer in Oklahoma, 1997-2012. J Okla State Med Assoc. 2016, 109:355365.

12. Howard SC, Metzger ML, Wilimas JA, Quintana Y, Pui CH, Robison LL, Ribeiro RC: Childhood cancer epidemiology in low-income countries. Cancer. 2008, 112:461-472. 10.1002/cncr.23205

13. National Cancer Institute: cancer in children and adolescents. (2018). Accessed: February 12, 2021: https://www.cancer.gov/types/childhood-cancers/child-adolescent-cancers-fact-sheet.

14. Williams LA, Richardson M, Marcotte EL, Poynter JN, Spector LG: Sex ratio among childhood cancers by single year of age. Pediatr Blood Cancer. 2019, 66:e27620. 10.1002/pbc.27620

15. Bourusly M, Obaid M, Bourhamah M: Children with Down syndrome and leukemia in Kuwait-experience of the only pediatric hematology oncology department in Kuwait. Cancer Reports Rev. 2017, 1:3-4. 10.15761/CRR.1000134

16. Ray A, Oliver TR, Halder P, Pal U, Sarkar S, Dutta S, Ghosh S: Risk of Down syndrome birth: consanguineous marriage is associated with maternal meiosis-II nondisjunction at younger age and without any detectable recombination error. Am J Med Genet A. 2018, 176:2342-2349. 10.1002/ajmg.a.40511

17. Mandalenakis Z, Karazisi C, Skoglund K, Rosengren A, Lappas G, Eriksson P, Dellborg M: Risk of cancer among children and young adults with congenital heart disease compared with healthy controls. JAMA Netw Open. 2019, 2:e196762. 10.1001/jamanetworkopen.2019.6762

18. Stjernfelt KJ, von Stedingk K, Wiebe T, Hjorth L, Olsson H, Øra I: Predominance of girls with cancer in families with multiple childhood cancer cases. BMC Cancer. 2017, 17:868. 10.1186/s12885-017-3899-8

19. Draper GJ, Sanders BM, Lennox EL, Brownbill PA: Patterns of childhood cancer among siblings. Br J Cancer. 1996, 74:152-158. 10.1038/bjc.1996.331

20. Efficient use of budget needed to enhance education's quality, ranking in Kuwait . (2017). Accessed: 


\section{Cureus}

February 12, 2021: https://www.pressreader.com/kuwait/kuwait-times/20170228/281573765474454.

21. Erdmann F, Winther JF, Dalton SO, et al.: Survival from tumours of the central nervous system in Danish children: is survival related to family circumstances?. Int J Cancer. 2018, 142:671-680. 10.1002/ijc.31082

22. Bray F, Ferlay J, Soerjomataram I, Siegel RL, Torre LA, Jemal A: Global cancer statistics 2018: GLOBOCAN estimates of incidence and mortality worldwide for 36 cancers in 185 countries. CA Cancer J Clin. 2018, 68:394-424. 10.3322/caac. 21492

23. Jawass MA, Al-Ezzi JI, Bin Gouth HS, Bahwal SA, Bamatraf FF, Ba'amer AA: Pattern of malignancies in children $<15$ years of age reported in Hadhramout Cancer Registry, Yemen between 2002 and 2014. Saudi Med J. 2016, 37:513-520. 10.15537/smj.2016.5.14954

24. Eapen M: Surviving childhood cancer: a sobering story. Blood. 2018, 131:2603-2604. 10.1182/blood-201805-847517

25. National Cancer Institute: SEER cancer statistics review, 1975-2017 . (2020). Accessed: November 10, 2020 : https://seer.cancer.gov/csr/1975_2017/.

26. Zhang AS, Ostrom OT, Kruchko C, Rogers L, Peereboom DM, Barnholtz-Sloan IS: Complete prevalence of malignant primary brain tumors registry data in the United States compared with other common cancers, 2010. Neuro Oncol. 2017, 19:726-735. 10.1093/neuonc/now252

27. Landier W, Armenian S, Bhatia S: Late effects of childhood cancer and its treatment . Pediatr Clin North Am. 2015, 62:275-300. 10.1016/j.pcl.2014.09.017

28. Rodriguez-Galindo C, Friedrich P, Alcasabas P, et al.: Toward the cure of all children with cancer through collaborative efforts: pediatric oncology as a global challenge. J Clin Oncol. 2015, 33:3065-3073. 10.1200/ICO.2014.60.6376

29. Ju HY, Moon EK, Lim J, Park BK, Shin HY, Won YJ, Park HJ: Second malignant neoplasms after childhood cancer: a nationwide population-based study in Korea. PLoS One. 2018, 13:e0207243.

10.1371/journal.pone.0207243

30. Stiller CA: Population based survival rates for childhood cancer in Britain, 1980-91 . BMJ. 1994, 309:16121616. $10.1136 / \mathrm{bmj} .309 .6969 .1612$ 